\title{
Influence of different sowing patterns on the productivity and water use efficiency of some lentil cultivar
}

\author{
Omnia S.M. Hashem ${ }^{1^{*}}$ and M.A. Ibrahim ${ }^{2}$ \\ ${ }^{1}$ Crop Physiology Research Department, Field Crops Research Institute, A.R.C., Giza, Egypt. \\ ${ }^{2}$ Food Legume Research Department, Field Crops Research Institute, A.R.C., Giza, Egypt. \\ *Corresponding Author: dromnia-hashem@hotmail.com
}

Received on: 6-7-2021

Accepted on: 2-9-2021

\begin{abstract}
A field experiment was conducted during two successive winter seasons of 2018/2019 and 2019/2020 at Giza Experimental Farm of the Agriculture Research Center, Giza, Egypt, to study the effect of different sowing pattems on the productivity and water use efficiency of some lentil cultivar. Three lentil cultivars i.e. Sinai 1, Giza 29 and Giza 51 were planted with different sowing patterns as follows: Broadcast, Drilling(Rows); on ridges Furrow $50 \mathrm{~cm}$, on ridges Furrow $75 \mathrm{~cm}$ and on ridges Furrow $100 \mathrm{~cm}$. The following traits were studied: (1) Growth characters (2) Yield and yield components, (3) Irrigation water use efficiency of lentil cultivars "IWUE ", and (4) Photosynthetic pigment of leaves and total soluble protein content of lentil seeds. The obtained results could be summarized as follows.

Lentil cv. "Giza 51" was recorded the highest values of all growth and yield components with all sowing pattems where as Sinai $1 \mathrm{cv}$. was recorded the lowest values of all the studied traits in both seasons. Also, the lowest amount of water consumptive use (WCU) was obtained from sowing method (Ridge-Furrow $75 \mathrm{~cm}$ ) followed by Ridge-Furrow 50 $\mathrm{cm}$ for the three cultivars compared to the traditional planting.

So we recommend cultivation of three lentil cultivars under Ridge-Furrow $75 \mathrm{~cm}$ cultivation methods.
\end{abstract}

KEYWORDS: Sowing patterns, Lentil cv., Productivity, Physiological studied, water relation.

\section{INTRODUCTION}

Lentil (Lens culinaris Medik.) is one of the most promising legume crops providing nutritional and food assurance to human beings (Mondal et al., 2013a) and high-quality lentil hay is extensively used as animal feed (Lardy and Anderson, 2009). Due to extensive production of lentil crop in rain-fed agriculture system, its growth and yield are mainly determined by the levels of precipitation. It also supports crop rotation due to its potential to sustain soil productivity by nitrogen fixation (Abi-Ghanem et al., 2011). The average yield of lentil in Egypt 2438 tons $^{-1}$ (FAO, 2020). Further, the area under lentil cultivation has been decreasing at a faster rate because of increasing demand for staple grain like wheat (Ali and Rahman, 2004). This situation is worsening day by day because of the poor yield of pulses compared to cereals. The major constraints responsible for low yield of lentil are short growth duration particularly the slow rate of dry matter accumulation prior to flowering and unfavorable canopy structure (Mondal et al., 2013b). Lentil seeds contain $1-2 \%$ fat, $24-32 \%$ proteins and minerals (iron, cobalt and iodine) as well as vitamins (lysine and arginine) (Kowieska and Petkov, 2003; ElZoghbi, 1998). Lentils are prepared in several methods including soaking, boiling, sprouting/germination, fermentation, frying and dry- heat methods. Other ways to benefit from it is processed lentil, lentil snacks and medicinal uses (Raghuvanshi and Singh 2009). On the other hand lentil vegetative parts can be used as green manure (Kara, 2008).

Lentils are considered as a moderately drought resistant crop but can be grown under irrigation with careful water management. Lentil plants do not like wet feet and therefore do not tolerate waterlogged soils, and will die if flooded. Excess water in lentil crop can cause problems including delayed maturity, increased disease, and lower yield. This crop is poorly competitive with weeds due to its small stature and slow growth early in the season (Ball, et al., 1997, McDonald, et al., 2007) or restricted by water deficit (Lal, et al., 1988). The growth could be improved by its sowing on the proper date (Ayoub Abodalla, 2014). Moreover, there are several causes responsible for low yield of lentil of which the use of traditional local cultivars, low plant density per unit area, weed infestation and poor crop management practices constitute the major ones. Use of the modern lentil cultivars and maintenance of proper plant density per unit area under suitable planting methods would thus help in increasing the yield from per unit area. Plant spacing affects plant grow th and yield due to increased competition with increased plant population. Moreover, the optimum plant population differs with the availability of soil 
moisture, relative humidity and nutrients. Higher plant population i.e. close plant spacing reduced plant growth and yield components Wanns et al., 1986 and Osman et al., 2010. So, spacing for line sowing is recommended to maintain the required number of plant population and undertake intercultural operations for harvesting higher yield. Most of the farmers follow broadcast method or line sowing method without maintaining proper spacing for growing lentil as a result reported by many researchers (Parveen and Bhuiya, 2010). So, Row planting was better than broadcast (Roysharma et al., 1984 and Nazir et al., 1992). Lopez-Bellido et al., 2005 reported that increasing plant space reduced weed competition and evaporation of water from the soil under the crop.

This investigation aimed to study the effect of different sowing patterns on the productivity and water use efficiency of some lentil cultivar to over watering and poor competitive against weeds.

\section{MATERIALS AND METHODS}

A field experiment was conducted during two successive winter seasons on $21^{\text {th }}$ November of 2018/2019 and 2019/2020. Three lentil genotypes with different genetic characters namely; Sinai 1,
Giza 29 and Giza 51 were used at Giza Experimental Farm of the Agriculture Research Center, Giza, Egypt.

Seeds were used at the rate of $45 \mathrm{~kg} \mathrm{fed}^{-1}$ and inoculated with Rhizobium as coating at the rate of $200 \mathrm{~g} / \mathrm{fad}$. Two irrigations were used the first was immediately after sowing and the second irrigation was added at three weeks after sowing. All agricultural practices were applied as recommended for lentil production in Giza region. The experiment treatments were arranged at split-plot design with three replicates. The main plots were randomly assigned for the five sowing pattern treatments, whereas lentil cultivars were distributed in sub plots.

The designed of five sowing patterns as follows:

1. Broadcast (Traditional).

2. Drilling (in rows, $25 \mathrm{~cm}$ apart).

3. On ridges Furrow $50 \mathrm{~cm}$ in hills $10 \mathrm{~cm}$ apart on the two sides of ridge.

4. On ridges Furrow $75 \mathrm{~cm}$ in hills at $10 \mathrm{~cm}$ apart on three rows of ridge.

5. On ridges Furrow $100 \mathrm{~cm}$ by broadc ast way $(1 \mathrm{~m}$ $\mathrm{x} 1 \mathrm{~m})$.

Name, pedigree and origin of the studied three lentil genotypes.

\begin{tabular}{ccc}
\hline Name & Pedigree & Origin \\
\hline Sinai -1 & Selection from Argentina variety precoz, early maturing & Argentina \\
Giza 29 & Landrace, high yield potential & ARC, Egypt \\
Giza 51 & Selection from hybrid family & ARC, Egypt \\
\hline
\end{tabular}

\subsection{Growth and growth analysis:}

Random sample of five plants from the second row of each plot was taken at 80 days after sowing (DAS), to determine: Plant height/plant $(\mathrm{cm})$, Shoot dry weight/plant (g), No of tiller / plant and Crop growth rate (CGR, g/week) was estimated for two growth periods of 65-80 and 80-95 DAS using the following formula according to Hunt (1990).

Where:

$$
\mathrm{CGR}=\left(\mathrm{W}_{2}-\mathrm{W}_{1}\right) /\left(\mathrm{T}_{2}-\mathrm{T}_{1}\right)
$$

$\mathrm{W}_{2}-\mathrm{W}_{1}$ : difference in plant dry matter accumulated between two successive samples.

$\mathrm{T}_{2}-\mathrm{T}_{1}$ : number of weeks between two successive samples.

\subsection{Chemical composition:}

2.2.1. Photosynthetic pigments assay: Chlorophyll was extracted by $85 \%$ acetone from fresh leaf sample at 80 DAS according to the method of Metzener et al. (1965). The concentration of the different pigment (total chlorophyll and carotenoids) w as determined and converted to $\mathrm{mg} / \mathrm{g}$.dry w eight.
2.2.2. At harvest time, the total soluble protein content of seeds was estimated quantitatively in the borate buffer extract using the method described by Bradford (1976). The protein content of seeds was calculated as $\mathrm{mg} / \mathrm{g}$.dry weight.

\subsection{Yield and its components:}

At harvest time (mid April), five individual plants were randomly taken to determine number of pods per plant, pods weight per plant (g), 100- seed weight $(\mathrm{g})$, seed number/plant and seed weight per plant $(\mathrm{g})$. Also, seed weight $/ \mathrm{m}^{2}(\mathrm{~g})$, straw yield /fad $(\mathrm{kg})$, seed yield/fad $(\mathrm{kg})$ and Harvest index $(\mathrm{HI})$.

$\mathrm{HI}(\%)=($ Seed yield/biological yield $) \times 100$

\subsection{Water relations:}

\subsubsection{Water consumptive use:}

Soil samples were taken, using a regular augar, at planting time, just before and 48 hours after each irrigation and at harvesting time for soil moisture determination. Duplicate of soil samples were taken from 0-150, 150-300, 300-450 and 450- 
Omnia S.M. Hashem and M.A. Ibrahim., 2021

$600 \mathrm{~mm}$ depths and their moisture contents were following Table.

\begin{tabular}{ccccc} 
gravimetric ally & determined and & presented in & & \\
\hline $\begin{array}{c}\text { Depth } \\
(\mathbf{m m})\end{array}$ & $\begin{array}{c}\text { Wilting point } \\
\mathbf{\%}\end{array}$ & $\begin{array}{c}\text { Field capacity } \\
\mathbf{\%}\end{array}$ & $\begin{array}{c}\text { Available water } \\
\mathbf{\%}\end{array}$ & $\begin{array}{c}\text { Bulk density } \\
\left(\mathbf{g} / \mathbf{c m}^{\mathbf{3}}\right)\end{array}$ \\
\hline $0-150$ & 16.33 & 32.70 & 16.37 & 1.15 \\
$150-300$ & 15.42 & 29.12 & 13.70 & 1.22 \\
$300-450$ & 15.10 & 26.71 & 11.61 & 1.20 \\
$450-600$ & 14.82 & 24.82 & 10.00 & 1.28 \\
\hline
\end{tabular}

The depleted soil moisture was detected after each irrigation and the following equation was used to calculating water consumptive use according to (Israelsen and Hansen, 1962):

$\mathrm{Cu}=\mathrm{D} \times \mathrm{Bd} \times\left(\mathrm{e}_{2}-\mathrm{e}_{1}\right) / 100$

where,

$\mathrm{Cu}$ is water consumptive use (ET) in $\mathrm{mm}$

$\mathrm{D}$ is soil depth (mm)

$\mathrm{Bd}$ is bulk density in $\mathrm{g} / \mathrm{cm}^{3}$

$\mathrm{e}_{1}, \mathrm{e}_{2}$ is soil moisture content before and after each irrigation.

\subsubsection{Water use efficiency (WUE):}

Water use efficiency $\left(\mathrm{Kg} / \mathrm{m}^{3} / \mathrm{fad}\right.$. $)$ was calculated for each treatment according to the equation described by Vites (1965) as follows: WUE = Seed yield $(\mathrm{Kg} / \mathrm{fad}) /$ seasonal water consumption in $\mathrm{m}^{3} / \mathrm{fad}$.

\subsection{Statistical analysis:}

Data of all parameters were analyzed using analysis of variance (ANOVA) according to the standard procedure of Snedec or and Cochran (1980) at $5 \%$ significance level and the means were compared by LSD test to check difference.

\section{RESULTS AND DISCUSSION}

\subsection{Growth and growth analysis}

Data in Table (1) showed that the highest values of plant height $(\mathrm{cm})$, No. of branches/ plant, shoot dry wt /plant and crop grow th rate at 65-80 and 8095 DAS were obtained under Ridge-Furrow $75 \mathrm{~cm}$ sowing method in the first and second seasons. Also, such traits significantly increased with cultivar Giza 51 followed by Giza 29. This increase in plant height could be justified on the bases of increase in number of plants per unit area coupled with high plant competition for light lead to taller plants as reported by Mahmoud (2014). Similar results were also reported by Habbasha et al. (1996) and Singh et al. (2003) stated that increasing plant density lead to increase in plant height.

Also, results showed that crop growth rate (CGR) was higher in the second period than in the first one that due to the accumulation of total dry matter (TDM) with time up to maturity. This increase may be due to the increase of moisture content in root zone, hence the plants will grow with lowest water stress and present of desirable distance (Abd El-Tawwab et al., 2007). Also, that based on the accumulation of dry matter resulted from photosynthetic compound, and then most of those compounds are transformed to seeds at the second period (pod formation) Edwards et al. (2005). Also data presented in Table (1) showed that the interaction effect between different sowing pattern and lentil cultivars was significant for plant height (cm), No. of branches/ plant, shoot dry wt /plant and crop growth rate and the maximum values obtained from treatment (Ridge-Furrow $75 \mathrm{~cm}$ sowing method X Giza 51 cultivar) in the two seasons under study.

\subsection{Photosynthetic pigment and Total soluble Protein:}

Photosynthetic pigments of leaves (chl $a+b$ and carotenoids) and protein content of seeds were increased under Ridge-Furrow $75 \mathrm{~cm}$ sowing method compared to other sowing method under study as shown in Table (2). Results indicated that Giza 51 gave the highest values of total chlorophyll and carotenoids as well as seed protein content than the other cultivars. This increase may be attributed to the majority of leaf $\mathrm{N}$ which accumulated in the chloroplast, where photosynthesis takes place, resulting in a strong association between plant photosynthesis (Evans, 1989). Also, it is reported that TDM production strongly correlated with chlorophyll content in leaves (Mondal et al., 2011b). Results were supported by the results obtained by Dutta and Mondal (1998) who reported that TDM increased with advanced plant age up to physiological maturity due to rapid growth of pods at later plant age. Also, plant spacing in the field is very important to facilitate aeration and light penetration into plant leaves for optimizing photosynthesis rate (Ouji et al., 2016). Total soluble protein increased in Giza 51 cultivar seeds related to Lentil is capable of fixing their own nitrogen during growing season (Sarker, et al., 2003 and McDonald, et al., 2007).

The interaction between lentil cultivars and sowing patterns recorded a significant effect on leaves pigment content and seed protein content. The maximum values of total chlorophyll, carotenoids and protein content in the two seasons 
Scientific Journal of Agricultural Sciences 3 (2): 105-115, 2021

Table 1. Plant height, shoot dry weight, number of branches, and crop growth rate of three lentil cultivars as affected by different sowing patterns.

\begin{tabular}{|c|c|c|c|c|c|c|c|c|c|c|c|}
\hline \multirow{3}{*}{\multicolumn{2}{|c|}{ Treatment }} & \multirow{2}{*}{\multicolumn{2}{|c|}{ Plant height $(\mathrm{cm})$}} & \multirow{2}{*}{\multicolumn{2}{|c|}{ Shoot dry wt/plant(g) }} & \multirow{2}{*}{\multicolumn{2}{|c|}{ No. of branches/plant }} & \multicolumn{4}{|c|}{ Crop Growth rate (CGR)(g/week) } \\
\hline & & & & & & & & \multicolumn{2}{|c|}{ 65-80 DAS } & \multicolumn{2}{|c|}{ 80- 95 DAS } \\
\hline & & $2018 / 19$ & $2019 / 20$ & 2018/19 & $2019 / 20$ & $2018 / 19$ & $2019 / 20$ & $2018 / 19$ & $2019 / 20$ & $2018 / 19$ & $2019 / 20$ \\
\hline \multicolumn{12}{|l|}{ Sowing patterns } \\
\hline \multicolumn{2}{|l|}{ Broadcasting } & 40.56 & 38.78 & 6.08 & 5.82 & 1.78 & 1.67 & 1.46 & 1.32 & 2.03 & 1.73 \\
\hline \multicolumn{2}{|l|}{ Row s (Drilling) } & 44.78 & 42.56 & 6.56 & 6.39 & 2.56 & 2.22 & 1.66 & 1.57 & 2.58 & 2.17 \\
\hline \multicolumn{2}{|c|}{ Ridge-Furrow $50 \mathrm{~cm}$} & 49.00 & 46.89 & 8.10 & 7.92 & 3.11 & 2.89 & 2.36 & 2.26 & 2.80 & 2.54 \\
\hline \multicolumn{2}{|c|}{ Ridge-Furrow $75 \mathrm{~cm}$} & 52.11 & 49.78 & 8.53 & 8.28 & 3.44 & 3.11 & 2.54 & 2.41 & 3.18 & 2.90 \\
\hline \multicolumn{2}{|c|}{ Ridge- furrow (1mx1m) } & 47.22 & 45.56 & 7.36 & 7.23 & 2.67 & 2.56 & 2.00 & 1.94 & 2.51 & 2.14 \\
\hline \multicolumn{2}{|c|}{$\mathbf{L S D}_{(0.05)}$} & 0.87 & 1.18 & 0.03 & 0.02 & 0.29 & 0.32 & 0.02 & 0.02 & 0.03 & 0.04 \\
\hline \multicolumn{12}{|l|}{ Cultivars } \\
\hline \multicolumn{2}{|l|}{ Sinai 1} & 40.60 & 38.67 & 6.17 & 5.91 & 2.07 & 1.80 & 1.56 & 1.43 & 2.27 & 1.92 \\
\hline \multicolumn{2}{|l|}{ Giza 29} & 47.80 & 45.60 & 7.42 & 7.27 & 2.80 & 2.60 & 2.01 & 1.94 & 2.73 & 2.35 \\
\hline \multicolumn{2}{|l|}{ Giza 51} & 51.80 & 49.87 & 8.40 & 8.20 & 3.27 & 3.07 & 2.44 & 2.34 & 2.85 & 2.62 \\
\hline \multicolumn{2}{|l|}{$\operatorname{LSD}_{(0.05)}$} & 0.75 & 1.13 & 0.02 & 0.02 & 0.30 & 0.30 & 0.02 & 0.02 & 0.02 & 0.03 \\
\hline \multirow{3}{*}{ Broadcasting } & Sinai 1 & 32.67 & 31.00 & 5.29 & 5.08 & 1.33 & 1.00 & 1.14 & 1.03 & 1.83 & 1.45 \\
\hline & Giza 29 & 43.33 & 42.00 & 6.23 & 6.12 & 2.00 & 2.00 & 1.50 & 1.45 & 1.86 & 1.58 \\
\hline & Giza 51 & 45.67 & 43.33 & 6.73 & 6.26 & 2.00 & 2.00 & 1.73 & 1.50 & 2.40 & 2.16 \\
\hline \multirow{3}{*}{ Row s (Drilling) } & Sinai 1 & 39.00 & 36.33 & 5.51 & 5.23 & 2.00 & 1.67 & 1.25 & 1.11 & 2.06 & 1.72 \\
\hline & Giza 29 & 46.00 & 43.67 & 6.55 & 6.43 & 2.67 & 2.33 & 1.62 & 1.56 & 2.83 & 2.41 \\
\hline & Giza 51 & 49.33 & 47.67 & 7.62 & 7.49 & 3.00 & 2.67 & 2.11 & 2.05 & 2.87 & 2.40 \\
\hline \multirow{3}{*}{$\begin{array}{l}\text { Ridge-Furrow } \\
50 \mathrm{~cm}\end{array}$} & Sinai 1 & 43.33 & 41.33 & 6.81 & 6.50 & 2.33 & 2.00 & 1.89 & 1.73 & 2.69 & 2.37 \\
\hline & Giza 29 & 49.67 & 47.00 & 8.33 & 8.10 & 3.00 & 3.00 & 2.43 & 2.31 & 2.87 & 2.50 \\
\hline & Giza 51 & 54.00 & 52.33 & 9.16 & 9.14 & 4.00 & 3.67 & 2.77 & 2.74 & 2.83 & 2.76 \\
\hline \multirow{3}{*}{$\begin{array}{l}\text { Ridge-Furrow } \\
75 \mathrm{~cm}\end{array}$} & Sinai 1 & 46.67 & 43.67 & 7.02 & 6.56 & 2.67 & 2.33 & 1.97 & 1.74 & 2.68 & 2.43 \\
\hline & Giza 29 & 52.00 & 50.33 & 8.52 & 8.36 & 3.33 & 3.00 & 2.48 & 2.40 & 3.42 & 3.02 \\
\hline & Giza 51 & 57.67 & 55.33 & 10.06 & 9.94 & 4.33 & 4.00 & 3.16 & 3.09 & 3.44 & 3.24 \\
\hline \multirow{3}{*}{$\begin{array}{l}\text { Ridge- furrow } \\
(1 \mathrm{mx} 1 \mathrm{~m})\end{array}$} & Sinai 1 & 41.33 & 41.00 & 6.21 & 6.19 & 2.00 & 2.00 & 1.56 & 1.55 & 2.11 & 1.64 \\
\hline & Giza 29 & 48.00 & 45.00 & 7.47 & 7.35 & 3.00 & 2.67 & 2.01 & 1.95 & 2.68 & 2.26 \\
\hline & Giza 51 & 52.33 & 50.67 & 8.41 & 8.16 & 3.00 & 3.00 & 2.43 & 2.30 & 2.73 & 2.53 \\
\hline \multicolumn{2}{|l|}{$\operatorname{LSD}_{(0.05)}$} & 1.69 & 2.52 & 0.04 & 0.03 & 0.67 & 0.67 & 0.07 & 0.07 & 0.08 & 0.11 \\
\hline
\end{tabular}


Table 2. Leaves content of Pigment and total soluble protein of seeds in three lentil cultivars as affected by different sowing patterns.

\begin{tabular}{|c|c|c|c|c|c|c|c|}
\hline \multirow{3}{*}{\multicolumn{2}{|c|}{ Treatment }} & \multicolumn{4}{|c|}{$\begin{array}{l}\text { Photosynthetic pigments of leaves } \\
\text { (mg/g.d.wt) }\end{array}$} & \multicolumn{2}{|c|}{$\begin{array}{l}\text { Protein content } \\
\text { mg/g.d.wt }\end{array}$} \\
\hline & & \multicolumn{2}{|c|}{ Total chlorophyll } & \multicolumn{2}{|c|}{ Carotenoides } & \multirow[b]{2}{*}{ 2018/19 } & \multirow[b]{2}{*}{$2019 / 20$} \\
\hline & & $2018 / 19$ & $2019 / 20$ & 2018/19 & $2019 / 20$ & & \\
\hline \multicolumn{8}{|l|}{ Sowing patterns } \\
\hline Broadcasting & & 2.34 & 2.17 & 0.50 & 0.46 & 7.22 & 6.94 \\
\hline Row s (Drilling) & & 2.76 & 2.52 & 0.58 & 0.53 & 7.31 & 7.09 \\
\hline Ridge-Furrow 5 & & 3.07 & 2.86 & 0.67 & 0.62 & 7.67 & 7.50 \\
\hline Ridge-Furrow 7 & & 3.23 & 2.99 & 0.72 & 0.66 & 7.90 & 7.68 \\
\hline Ridge- furrow ( & x1m) & 2.87 & 2.70 & 0.62 & 0.58 & 7.55 & 7.29 \\
\hline $\operatorname{LSD}_{(0.05)}$ & & 0.03 & 0.02 & 0.01 & 0.01 & 0.05 & 0.02 \\
\hline \multicolumn{8}{|l|}{ Cultivars } \\
\hline Sinai 1 & & 1.84 & 1.77 & 0.51 & 0.47 & 6.51 & 6.28 \\
\hline Giza 29 & & 2.69 & 2.47 & 0.60 & 0.57 & 7.67 & 7.44 \\
\hline Giza 51 & & 4.03 & 3.70 & 0.74 & 0.68 & 8.41 & 8.19 \\
\hline \multicolumn{8}{|l|}{ Interaction } \\
\hline \multirow[t]{3}{*}{ Broadcasting } & Sinai 1 & 1.47 & 1.37 & 0.39 & 0.36 & 6.48 & 6.10 \\
\hline & Giza & 2.30 & 2.18 & 0.52 & 0.49 & 7.04 & 6.78 \\
\hline & Giza & 3.26 & 2.95 & 0.60 & 0.55 & 8.14 & 7.92 \\
\hline \multirow[t]{3}{*}{ Row s (Drilling) } & Sinai 1 & 1.50 & 1.45 & 0.48 & 0.43 & 6.48 & 6.13 \\
\hline & Giza 29 & 2.75 & 2.40 & 0.54 & 0.51 & 7.31 & 7.09 \\
\hline & Giza 51 & 4.05 & 3.71 & 0.71 & 0.65 & 8.14 & 8.05 \\
\hline Ridge-Furrow & Sinai 1 & 2.17 & 2.04 & 0.57 & 0.50 & 6.48 & 6.38 \\
\hline \multirow{2}{*}{$50 \mathrm{~cm}$} & Giza 29 & 2.81 & 2.61 & 0.64 & 0.62 & 8.01 & 7.83 \\
\hline & Giza 51 & 4.23 & 3.95 & 0.78 & 0.75 & 8.52 & 8.29 \\
\hline Ridge-Furrow & Sinai 1 & 2.30 & 2.25 & 0.62 & 0.59 & 6.62 & 6.41 \\
\hline \multirow[t]{2}{*}{$75 \mathrm{~cm}$} & Giza 29 & 2.82 & 2.62 & 0.66 & 0.63 & 8.28 & 8.11 \\
\hline & Giza 51 & 4.57 & 4.10 & 0.88 & 0.76 & 8.79 & 8.53 \\
\hline Ridge- furrow & Sinai 1 & 1.78 & 1.75 & 0.49 & 0.46 & 6.48 & 6.36 \\
\hline \multirow{3}{*}{$(1 \mathrm{mx} 1 \mathrm{~m})$} & Giza 29 & 2.79 & 2.56 & 0.64 & 0.61 & 7.73 & 7.38 \\
\hline & Giza 51 & 4.05 & 3.80 & 0.73 & 0.68 & 8.45 & 8.14 \\
\hline & & $\mathbf{0 . 0 7}$ & $\mathbf{0 . 0 7}$ & 0.01 & 0.02 & 0.06 & 0.02 \\
\hline \multirow{2}{*}{\multicolumn{4}{|c|}{$\begin{array}{l}\text { was obtained by (Ridge-Furrow } 75 \mathrm{~cm} \text { sowing } \\
\text { method X Giza } 51 \text { cultivars), followed by Giza } 29 \\
\text { and Sinail under the same planting method. While, } \\
\text { the lowest values were obtained under (broadcast } \\
\text { planting methods x Sinail) treatment. These results } \\
\text { are agree with those obtained by Wells (1993) who } \\
\text { reported that plant spacing affects leaf area, light } \\
\text { interception and canopy apparent photosynthesis. } \\
\text { Edwards et al. (2005) and Boquet (1990) reported } \\
\text { that light energy efficiency in photosynthesis } \\
\text { depends on the plant density and transition of light } \\
\text { to plant canopy. }\end{array}$}} & \multirow{4}{*}{\multicolumn{4}{|c|}{$\begin{array}{l}\text { and } 26.89 \% \text { respectively) in first season and }(3.63 \mathrm{~g} \text {, } \\
4.87 \mathrm{~g}, 101.11,6.99 \mathrm{~g}, 127.02 \mathrm{~g}, 533.50 \mathrm{Kg} \text {, } \\
1344.31 \mathrm{Kg} \text { and } 26.50 \% \text { respectively) in second } \\
\text { season on all measured parameters. } \\
\text { With respect of cultivars, the highest } 100 \text {-seed } \\
\mathrm{wt} \text {, seed wt/plant, No. of pods/plant, pods wt/plant, } \\
\text { seeds wt } / \mathrm{m}^{2} \text {, seed yield/fad, straw yield/fad and } \\
\text { Harvest index (HI) were observed in Giza } 51(3.48 \\
\mathrm{g}, 4.95 \mathrm{~g}, 97.67,7.09 \mathrm{~g}, 114.46 \mathrm{~g}, 480.72 \mathrm{Kg} \text {, } \\
1226.87 \mathrm{Kg} \text { and } 27.90 \% \mathrm{respectively)} \text { in first season } \\
\text { and }(3.42 \mathrm{~g}, 4.81 \mathrm{~g}, 90.60,6.82 \mathrm{~g}, 110.42 \mathrm{~g} \text {, } \\
463.78 \mathrm{Kg}, 1173.57 \mathrm{Kg} \text { and } 27.63 \% \text { respectively) in } \\
\text { second season. That followed by Giza } 29 \text { cultivar. } \\
\text { Data are in harmony with those obtained by } \\
\text { Singh and Verma (1996) who found that line space } \\
\text { methods increased yield characters. This can be } \\
\text { explain by the fact that, the increment of yield is due } \\
\text { to the increase of branches number and plant height } \\
\text { resulted by the effect of ridging, where the desirable } \\
\text { distance is existed. Also, number of pods/plant was } \\
\text { affected significantly by different row spacing, so } \\
\text { that pods number per plant increased with increasing }\end{array}$}} \\
\hline & & & & & & & \\
\hline \multicolumn{4}{|c|}{ 3.3. Yield and yield components: } & & & & \\
\hline \multicolumn{4}{|c|}{$\begin{array}{l}\text { Variation in yield and yield components of the } \\
\text { planting methods and cultivars were significant } \\
\text { (Table } 3 \text { and } 4 \text { ). Maximum values of } 100 \text {-seed wt, } \\
\text { seed wt/plant, No. of pods/plant, pods wt } / \text { plant, } \\
\text { seeds wt } / \mathrm{m}^{2} \text {, seed yield/fad, straw yield/fad and } \\
\text { Harvest index (HI) were obtained from (Ridge- } \\
\text { Furrow } 75 \mathrm{~cm} \text { sowing method) treatment }(3.75 \mathrm{~g} \text {, } \\
4.99 \mathrm{~g}, 109,7.14 \mathrm{~g}, 131.21 \mathrm{~g}, 551.08 \mathrm{Kg}, 1403.89 \mathrm{Kg}\end{array}$} & & & & \\
\hline
\end{tabular}


Table 3. Some yield component of three lentil cultivars as affected by different sowing patterns.

\begin{tabular}{|c|c|c|c|c|c|c|c|c|c|}
\hline \multirow{2}{*}{\multicolumn{2}{|c|}{ Treatment }} & \multicolumn{2}{|c|}{ 100-seed wt (g) } & \multicolumn{2}{|c|}{ Seed wt/plant (g) } & \multicolumn{2}{|c|}{ No. of pods/plant } & \multicolumn{2}{|c|}{ Pods wt /plant(g) } \\
\hline & & $2018 / 19$ & $2019 / 20$ & $2018 / 19$ & $2019 / 20$ & $2018 / 19$ & $2019 / 20$ & $2018 / 19$ & $2019 / 20$ \\
\hline \multicolumn{10}{|c|}{ Sowing patterns } \\
\hline \multicolumn{2}{|c|}{ Broadcasting } & 2.94 & 2.84 & 2.69 & 2.55 & 57.44 & 51.89 & 4.61 & 4.33 \\
\hline \multicolumn{2}{|c|}{ Rows (Drilling) } & 3.13 & 2.97 & 3.42 & 3.32 & 73.44 & 68.22 & 5.22 & 4.97 \\
\hline \multicolumn{2}{|c|}{ Ridge-Furrow $50 \mathrm{~cm}$} & 3.50 & 3.43 & 4.74 & 4.60 & 101.77 & 91.78 & 6.64 & 6.41 \\
\hline \multicolumn{2}{|c|}{ Ridge-Furrow $75 \mathrm{~cm}$} & 3.75 & 3.63 & 4.99 & 4.87 & 109.00 & 101.11 & 7.14 & 6.99 \\
\hline \multicolumn{2}{|c|}{ Ridge- furrow (1mx1m) } & 3.34 & 3.26 & 4.07 & 3.95 & 83.44 & 80.67 & 6.06 & 5.86 \\
\hline \multicolumn{2}{|c|}{$\mathbf{L S D}_{(0.05)}$} & 0.02 & 0.01 & 0.01 & 0.02 & 1.92 & 1.47 & 0.02 & 0.02 \\
\hline \multicolumn{10}{|l|}{ Cultivars } \\
\hline \multicolumn{2}{|l|}{ Sinai 1} & 3.17 & 3.02 & 2.53 & 2.41 & 68.60 & 64.07 & 4.74 & 4.58 \\
\hline \multicolumn{2}{|l|}{ Giza 29} & 3.34 & 3.24 & 4.47 & 4.35 & 88.80 & 81.53 & 5.96 & 5.73 \\
\hline \multicolumn{2}{|l|}{ Giza 51} & 3.48 & 3.42 & 4.95 & 4.81 & 97.67 & 90.60 & 7.09 & 6.82 \\
\hline \multirow{2}{*}{\multicolumn{2}{|c|}{$\begin{array}{l}\text { LSD }_{(0.05)} \\
\text { Interaction }\end{array}$}} & 0.01 & 0.01 & 0.05 & 0.02 & 1.72 & 0.91 & 0.01 & 0.02 \\
\hline & & & & & & & & & \\
\hline \multirow{3}{*}{ Broadcasting } & Sinai 1 & 2.74 & 2.53 & 1.67 & 1.53 & 48.00 & 43.67 & 3.64 & 3.41 \\
\hline & Giza 29 & 2.99 & 2.95 & 3.03 & 2.96 & 60.00 & 54.00 & 4.75 & 4.45 \\
\hline & Giza 51 & 3.09 & 3.05 & 3.37 & 3.16 & 64.33 & 58.00 & 5.43 & 5.13 \\
\hline \multirow{3}{*}{$\begin{array}{l}\text { Row s } \\
\text { (Drilling) }\end{array}$} & Sinai 1 & 2.96 & 2.64 & 2.06 & 1.92 & 53.33 & 48.33 & 4.27 & 4.06 \\
\hline & Giza 29 & 3.11 & 3.04 & 3.70 & 3.61 & 78.00 & 69.67 & 5.53 & 5.22 \\
\hline & Giza 51 & 3.32 & 3.24 & 4.50 & 4.44 & 89.00 & 86.67 & 5.86 & 5.63 \\
\hline Ridge- & Sinai 1 & 3.37 & 3.30 & 3.30 & 3.23 & 82.00 & 76.00 & 5.41 & 5.17 \\
\hline Furrow & Giza 29 & 3.49 & 3.45 & 5.26 & 5.14 & 105.33 & 95.67 & 6.37 & 6.13 \\
\hline $50 \mathrm{~cm}$ & Giza 51 & 3.64 & 3.54 & 5.67 & 5.44 & 118.00 & 103.67 & 8.15 & 7.93 \\
\hline Ridge- & Sinai 1 & 3.58 & 3.48 & 3.39 & 3.27 & 93.00 & 88.33 & 5.82 & 5.78 \\
\hline Furrow & Giza 29 & 3.77 & 3.54 & 5.75 & 5.59 & 111.67 & 102.00 & 7.14 & 7.03 \\
\hline $75 \mathrm{~cm}$ & Giza 51 & 3.92 & 3.89 & 5.82 & 5.75 & 122.33 & 113.00 & 8.45 & 8.17 \\
\hline Ridge- & Sinai 1 & 3.19 & 3.15 & 2.25 & 2.11 & 66.67 & 64.00 & 4.56 & 4.50 \\
\hline furrow & Giza 29 & 3.37 & 3.24 & 4.59 & 4.47 & 89.00 & 86.33 & 6.03 & 5.84 \\
\hline$(1 \mathrm{mx} 1 \mathrm{~m})$ & Giza 51 & 3.47 & 3.37 & 5.36 & 5.27 & 94.67 & 91.67 & 7.58 & 7.24 \\
\hline $\mathbf{L S D}_{(0.05)}$ & & 0.02 & 0.02 & 0.14 & 0.04 & 0.96 & 0.53 & 0.02 & 0.04 \\
\hline
\end{tabular}

the row spacing. Results revealed that increasing space led to increase No. of branch/plant which led to increase No. of pods/plant and pods wt/plant. Similarly, Momoh and Zhou (2001) stated that the higher branching observed in wide row spacing which increased number of pods per plant and pods wt per plant. This can be explained by the dominant effect of terminal bud lessens at lower densities and plants produce more auxiliary branches. So, they have better conditions for utilizing environmental conditions and produce moreflower.

Our results are consistent with results of Seyyed et al. (2014) and Idris (2008) on lentil, who indicated that increasing plant spacing increased number of pods per plant and consequently gave the highest seed yield.

When plants are widely spaced, biological yields tend to increase linearly with increased in plant spacing due to no or minimum competition between adjoining plants. Singh et al. (2003) reported that increased 100-seed weight due to the increase of pods number and seeds per plant as confirmed by Stoilova and Pereira (1999) and Sharar et al., 2001.

The lowest values of all yield components were obtained with Broadcasting and Rows (drilling) planting methods for all cultivars under study for two seasons.

The interaction between the two factors under study indicated that significant effect obtained from (ridge furrow $75 \mathrm{~cm}$ x Giza 51) for two seasons followed by Giza 29 cultivar under the same sowing method.

In contrast, the lowest yield and yield contributing characters were recorded under broadc ast traditional methods for the three cultivars. It might be due to difficulty to overcome weed lead to low seed yield in broadc ast planting method.

Results showed that an increase in row spacing led to higher seed yields per fad. The seeds wt per plant is closely correlated with the number of pods per plant, and is, therefore, an important yield component. Seed wt per plant increased when row spacing increased. Straw yield is sum of total dry 
Omnia S.M. Hashem and M.A. Ibrahim., 2021

Table 4. Some yield component of three lentil cultivars as affected by different sowing patterns.

\begin{tabular}{|c|c|c|c|c|c|c|c|c|c|}
\hline \multirow{2}{*}{\multicolumn{2}{|c|}{ Treatment }} & \multicolumn{2}{|c|}{ Seeds wt $/ \mathbf{m}^{2}(g)$} & \multicolumn{2}{|c|}{$\begin{array}{c}\text { Seed yield } \\
\text { /fad(kg) }\end{array}$} & \multicolumn{2}{|c|}{$\begin{array}{c}\text { Straw yield } \\
\text { /fad(Kg) }\end{array}$} & \multicolumn{2}{|c|}{ Harvest index \% } \\
\hline & & $2018 / 19$ & $2019 / 20$ & $2018 / 19$ & $2019 / 20$ & $2018 / 19$ & $2019 / 20$ & $2018 / 19$ & $2019 / 20$ \\
\hline \multicolumn{10}{|c|}{ Sowing patterns } \\
\hline \multicolumn{2}{|c|}{ Broadcasting } & 75.87 & 72.96 & 318.67 & 306.44 & 686.00 & 660.96 & 23.14 & 22.87 \\
\hline \multicolumn{2}{|c|}{ Row s (Drilling) } & 91.71 & 85.55 & 385.20 & 359.32 & 836.88 & 817.52 & 24.77 & 4.17 \\
\hline \multicolumn{2}{|c|}{ Ridge-Furrow $50 \mathrm{~cm}$} & 120.29 & 115.19 & 505.22 & 483.79 & 1148.78 & 1085.00 & 26.29 & 25.90 \\
\hline \multicolumn{2}{|c|}{ Ridge-Furrow $75 \mathrm{~cm}$} & 131.21 & 127.02 & 551.08 & 533.50 & 1403.89 & 1344.31 & 26.89 & 26.50 \\
\hline \multicolumn{2}{|c|}{ Ridge- furrow (1mx1m) } & 107.66 & 103.25 & 452.16 & 433.65 & 1031.33 & 977.90 & 25.48 & 24.57 \\
\hline \multicolumn{2}{|c|}{$\mathbf{L S D}_{(0.05)}$} & 0.64 & 0.53 & 0.90 & 0.24 & 2.58 & 8.23 & 0.48 & 0.17 \\
\hline \multicolumn{10}{|l|}{ Cultivars } \\
\hline \multicolumn{2}{|l|}{ Sinai 1} & 92.29 & 87.74 & 387.63 & 368.55 & 628.60 & 619.78 & 20.75 & 19.94 \\
\hline \multicolumn{2}{|l|}{ Giza 29} & 109.30 & 21 & 459.05 & 437.70 & 1208.67 & 1138.06 & 27 & 26.85 \\
\hline \multicolumn{2}{|l|}{ Giza 51} & 114.46 & 110.42 & 480.72 & 463.78 & 1226.87 & 1173.57 & 27.90 & 27.63 \\
\hline \multicolumn{2}{|l|}{$\begin{array}{l}\mathbf{L S D}_{(0.05)} \\
\text { Interaction }\end{array}$} & 21 & (A) 25 & 0.25 & 0.47 & 2.73 & 5.38 & ( 31 & 0.10 \\
\hline \multirow{3}{*}{ Broadcasting } & & 6 & & 288.86 & 273.04 & 326.67 & 323.17 & & 46 \\
\hline & Giza 29 & 77.55 & 73.48 & 325.73 & 308.61 & 847.00 & 776.53 & 25.61 & 25.08 \\
\hline & Giza 51 & 81.29 & 80.40 & 341.42 & 337.68 & 884.33 & 883.17 & 26.19 & 26.06 \\
\hline \multirow{3}{*}{$\begin{array}{l}\text { Row s } \\
\text { (Drilling) }\end{array}$} & Sinai 1 & 78.11 & 70.54 & 328.06 & 296.29 & 443.33 & 440.07 & 19.05 & 18.26 \\
\hline & Giza 29 & 98.16 & 90.50 & 412.26 & 380.09 & 984.67 & 960.17 & 27.13 & 26.44 \\
\hline & Giza 51 & 98.88 & 95.62 & 415.28 & 401.58 & 1082.67 & 1052.33 & 28.13 & 27.81 \\
\hline Ridge- & Sinai 1 & 103.33 & 98.81 & 433.99 & 414.99 & 844.67 & 828.33 & 22.74 & 22.31 \\
\hline Furrow & Giza 29 & 124.83 & 119.88 & 524.30 & 503.52 & 1225.00 & 1108.33 & 27.76 & 27.39 \\
\hline $50 \mathrm{~cm}$ & Giza 51 & 132.71 & 126.88 & 557.38 & 532.88 & 1376.67 & 1318.33 & 28.38 & 28.01 \\
\hline Ridge- & Sinai 1 & 114.54 & 110.88 & 481.06 & 465.68 & 898.33 & 891.33 & 23.70 & 23.22 \\
\hline Furrow & Giza 29 & 137.92 & 131.87 & 579.28 & 553.84 & 1796.67 & 1782.67 & 28.34 & 28.01 \\
\hline $75 \mathrm{~cm}$ & Giza 51 & 141.17 & 138.33 & 592.90 & 580.98 & 1516.67 & 1358.93 & 28.64 & 28.28 \\
\hline Ridge- & Sinai 1 & 96.71 & 93.51 & 406.19 & 392.74 & 630.00 & 616.00 & 20.64 & 18.42 \\
\hline furrow & Giza 29 & 108.02 & 105.34 & 453.67 & 442.43 & 1190.00 & 1062.60 & 27.66 & 27.33 \\
\hline$(1 \mathrm{mx} 1 \mathrm{~m})$ & Giza 51 & 118.25 & 110.90 & 496.64 & 465.79 & 1274.00 & 1255.10 & 28.15 & 27.96 \\
\hline $\mathbf{L S D}_{(0.05)}$ & & 0.27 & 0.78 & 0.52 & 0.30 & 7.90 & 7.90 & 0.29 & 0.09 \\
\hline
\end{tabular}

matter produced through physiological and biochemical processes occurring in the plant system. Parveen and Bhuiya (2010) reported that seeds rate is one of the main factors that have an important role on growth, yield and quality of lentil. An optimum spacing can ensure proper growth of the aerial and underground parts of the plant through efficient utilization of solar radiation, nutrients, water, land as well as air spaces. Spacing for line sowing is recommended to maintain the required number of plant population and to undertake intercultural operations for harvesting a higher yield.

\subsection{Water relations:}

\subsubsection{Seasonal water consumptive use (WCU):}

Seasonal water consumptive by three cultivars of lentil plants under different planting methods are presented in Table (5). Results indicated that the values of WCU for lentil plants ranged from 125.89 to $406.77 \mathrm{~m}^{3} /$ fad with respect to the mean of both seasons under study. Results revealed that the maximum value of WCU was achieved under Broadcast sowing method $\left(404.27 \mathrm{~m}^{3} / \mathrm{fad}\right)$ followed by Drilling sowing method $\left(247.16 \mathrm{~m}^{3} / \mathrm{fad}\right)$, however the low est value of WCU was obtained from ridge furrow $75 \mathrm{~cm}$ sowing method $\left(126.11 \mathrm{~m}^{3} / \mathrm{fad}\right)$. It is interesting to mention that the decrease of WCU could be arranged as the following descending order, Broadcasting > Rows (drilling) $>$ Ridge-furrow $(1 \mathrm{mx} 1 \mathrm{~m})>$ Ridge-furrow $(50 \mathrm{~cm})>$ Ridge furrow $(75 \mathrm{~cm})$. Such decrease of WCU due to the decrease of evapotranspiration under Ridge-furrow $75 \mathrm{~cm}$ sowing method. In this connection (Ibrahim ,1981) showed that the increase in evapotranspiration rate by maintain soil moisture at high level can be attributed to excess available water in the root zone to be consumed by the plant.

Regarding the difference between Lentil cultivars for WCU data of (Table 5) revealed that the maximum value was obtained by Giza 29 (231.86 $\mathrm{m}^{3} / \mathrm{fad}$ ), whereas the lowest value was gave by Sinai $1\left(230.14 \mathrm{~m}^{3} / \mathrm{fad}\right)$. The lowest value of seasonal 
Table 5. Seasonal water consumptive and water use efficiency as affected by different sowing patterns for three lentil cultivars.

\begin{tabular}{|c|c|c|c|c|c|c|c|}
\hline \multirow[t]{2}{*}{ Treatment } & & \multicolumn{3}{|c|}{$\begin{array}{c}\text { Seasonal water consumptive use } \\
\left(\mathrm{WCU}, \mathbf{~ m}^{3} / \mathbf{f a d} \text {.) }\right.\end{array}$} & \multicolumn{3}{|c|}{$\begin{array}{l}\text { Water use efficiency } \\
\text { (WUE, } \mathrm{Kg} / \mathrm{m}^{3} / \text { fad) }\end{array}$} \\
\hline & & $2018 / 19$ & $2019 / 20$ & Mean & $2018 / 19$ & $2019 / 20$ & Mean \\
\hline \multirow[t]{2}{*}{ Sowing patterns } & cultivars & & & & & & \\
\hline & Sinai 1 & 393.52 & 410.34 & 401.93 & 0.73 & 0.66 & 0.70 \\
\hline \multirow[t]{2}{*}{ Broadcasting } & Giza 29 & 397.87 & 415.66 & 406.77 & 0.82 & 0.74 & 0.78 \\
\hline & Giza 51 & 395.93 & 412.31 & 404.12 & 0.86 & 0.82 & 0.84 \\
\hline \multirow[t]{2}{*}{ Mean } & & 395.77 & 412.77 & 404.27 & 0.80 & 0.74 & 0.77 \\
\hline & Sinai 1 & 242.72 & 248.23 & 245.48 & 1.35 & 1.19 & 1.27 \\
\hline \multirow[t]{2}{*}{ Rows (Drilling) } & Giza 29 & 246.67 & 251.52 & 249.10 & 1.67 & 1.51 & 1.59 \\
\hline & Giza 51 & 244.70 & 249.11 & 246.91 & 1.70 & 1.61 & 1.66 \\
\hline Mean & & 244.70 & 249.62 & 247.16 & 1.57 & 1.44 & 1.51 \\
\hline \multirow{3}{*}{$\begin{array}{l}\text { Ridge-Furrow } 50 \\
\text { cm }\end{array}$} & Sinai 1 & 180.94 & 185.57 & 183.26 & 2.40 & 2.24 & 2.32 \\
\hline & Giza 29 & 179.75 & 182.34 & 181.05 & 2.92 & 2.76 & 2.84 \\
\hline & Giza 51 & 180.34 & 184.98 & 182.66 & 3.09 & 2.88 & 2.99 \\
\hline Mean & & 180.34 & 184.30 & 182.32 & 2.80 & 2.63 & 2.72 \\
\hline \multirow{3}{*}{$\begin{array}{l}\text { Ridge-Furrow } 75 \\
\text { cm }\end{array}$} & Sinai 1 & 124.50 & 127.43 & 125.97 & 3.86 & 3.65 & 3.76 \\
\hline & Giza 29 & 125.36 & 127.58 & 126.47 & 4.62 & 4.34 & 4.48 \\
\hline & Giza 51 & 124.28 & 127.50 & 125.89 & 4.77 & 4.56 & 4.67 \\
\hline Mean & & 124.71 & 127.50 & 126.11 & 4.42 & 4.18 & 4.30 \\
\hline \multirow{3}{*}{$\begin{array}{l}\text { Ridge- furrow } \\
\text { (1mx1m) }\end{array}$} & Sinai 1 & 190.02 & 198.11 & 194.07 & 2.14 & 1.98 & 2.06 \\
\hline & Giza 29 & 191.15 & 200.63 & 195.89 & 2.37 & 2.21 & 2.29 \\
\hline & Giza 51 & 190.54 & 199.42 & 194.98 & 2.61 & 2.34 & 2.48 \\
\hline Mean & & 190.57 & 199.39 & 194.98 & 2.37 & 2.18 & 2.28 \\
\hline \multirow{3}{*}{$\begin{array}{l}\text { General mean of } \\
\text { cultivars }\end{array}$} & Sinai 1 & 226.34 & 233.94 & 230.14 & 2.10 & 1.94 & 2.02 \\
\hline & Giza 29 & 228.16 & 235.55 & 231.86 & 2.48 & 2.31 & 2.40 \\
\hline & Giza 51 & 227.16 & 234.66 & 230.91 & 2.61 & 2.44 & 2.53 \\
\hline
\end{tabular}

water consumptive use (WCU) by Sinai 1 could be explained on the bases that this cultivars had the plant height $(40.60$ and $38.67 \mathrm{~cm})$ as well as number of branches/plant (2.07 and 1.80) in the first and second season, respectively as shown in (Table 1). These results are in line with those reported by Kassab et al., (2014).

The interaction between the two factors i.e different sowing patterns and Lentil cultivars on WCU data of Table (5) showed that the heighst value was obtained from (Broadcasting X Giza 29) which recorded $\left(406.77 \mathrm{~m}^{3} / \mathrm{fad}\right)$ whereas the lowest one recorded from (Ridge-furrow $75 \mathrm{~cm} \mathrm{X} \mathrm{Giza} \mathrm{51)}$ which recorded $\left(125.89 \mathrm{~m}^{3} / \mathrm{fad}\right)$.

\subsubsection{Water use efficiency (WUE):}

Water use efficiency (WUE) by lentil plants expressed as $\mathrm{kg}$ seeds produced per $\mathrm{m} 3$ of water consumed in complete evapotranspiration are presented in Table (5). WUE recorded the maximum value when lentil plants planted under ridge-furrow $75 \mathrm{~cm}$ sowing method $(4.30 \mathrm{Kg} / \mathrm{m} 3 / \mathrm{fad})$, these results may be explained due to the higher seed yield/fad and the low water consumed by such treatment. In this connection, Vites, (1965) concluded that WUE is not clearly depend on the water available and evapotranspiration limit, even the crop yield and the opportunity to increase it do depend on the adequacy of water supply.

Concerning the differences between lentil cultivars under study with respect to the values of WUE, results of Table (5) show that the highest value of WUE was obtained by Giza 51 (2.53 $\mathrm{Kg} / \mathrm{m} 3 / \mathrm{fad})$ followed by Giza $29(2.40 \mathrm{Kg} / \mathrm{m} 3 / \mathrm{fad})$. Such results indicated that Giza 51 gave the higher seed yield more than the increase in water consumed by the same lentil plant.

As for the effect of sowing patterns and lentil cultivars on WUE, results of Table (5) revealed that the maximum value of WUE was obtained when Giza 51 sown under Ridge-furrow $75 \mathrm{~cm}$ sowing $\operatorname{method}\left(4.67 \mathrm{Kg} / \mathrm{m}^{3} / \mathrm{fad}\right)$.

\section{CONCLUSION}

From the above result it is appears that apparently ridge furrow $75 \mathrm{~cm}$ sowing method was better than broadcast traditional methods. So our recommendation is use ridge furrow $75 \mathrm{~cm}$ planting method with Giza 51 lentil to improve yield and yield components as well as can overcome weed infestation and over flooded irrigation (sensitivity to water) than traditional planting methods. In addition, WUE increased when Giza 51 planting under RidgeFurrow $75 \mathrm{~cm}$ sowing method. 


\section{REFERENCE}

Abd El-Tawwab IM, Badawy ME, El-Khawaga S (2007). Developing and performance evaluation of a locally fabricated sugar beet planter. Misr J. Agric. Eng., 24(4): 648 - 665.

Abi-Ghanem R, Carpenter BL, Smith JL (2011). Cultivar effects on nitrogen fixation in peas and lentils. Biology \& Fertility Soils, 47(1):115-120.

Ali A, Rahman MM (2004). Selection criterion for selecting high yielding genotypes of lentil. Comm. Soil Sci. Plant Anal., 35: 1117-1122.

Ayoub Abdalla MH (2014). Effects of sowing date and plant population on Snap Bean (Phaseolus vulgaris $\mathrm{L}$.) growth and pod yield in Khartoum state. Universal Journal of Agricultural, 2(3): 115-118.

Ball DA, Ogg AG, Chevalier PM (1997). The influence of seeding rate on weed control in smallred lentil (Lens culinaris). Weed Sci. 45: 296-300.

Boquet DJ (1990). Plant population density and row spacing effects on soybean at post-optimal planting dates. Agron. J. 82: 59-64.

Bradford MM (1976). A rapid and sensitive method for quantitation of microgram quantities of protein utilization the principle of protein-dye binding. Annals of Biochem. 72:248-254.

Dutta RK, Mondal MMA (1998). Evaluation of lentil genotypes in relation to growth characteristics, assimilate distribution and yield potential. LENS Newsl., 25: 51-55.

Edwards JT, Purcell LC, Karcher DE (2005). Soybean yield and biomass to increasing plant population among diverse maturity groups. II. Light interception and utilization. Crop Sci., 45: 17781785.

El-Zoghbi M (1998). Nutritional quality of some protein sources. Annals of Agricultural Science, 36:2329-2339.

Evans JR (1989). Photosynthesis and nitrogen relationships in leaves of C3 plants. Oecologia, 78(1):9-19.

FAO (2020). Faostat, Fao Statistical Database. Retrievedfrom

http://www.fao.org/faostat/en/\#data/QC.

Habbasha KM, Adam SM, Rizk FA (1996). Growth and yield of pea (Pisum sativum) plant affected by plant density and foliar potassium applic ation. Egyptian J. Hort. 123(1):33-51.

Hunt R (1990). Basic Growth Analysis. Published by the Academic Division of Unwin Hyman Ltd., London. 55-72.

Ibrahim MA (1981). Evaluation of different methods for calculation potential evapotranspiration in North Delta. Ph.D. Thesis, Fac. Agric. Alex. Univ.

Idris AY (2008). Effect of seed size and plant spacing on yield and yield components of faba bean
(Viciafaba L.). Res. J. Agri. \& Biol. Sci., 4(2): 146148.

Israelsen OW, Hansen VE (1962). Irrigation principles and practices. The $3^{\text {rd }}$ edit, John Willey and Sons Inc., New York.

Kara K (2008). Field crops. Ataturk University, Faculty of Agricultural Engineering, Erzurum, Turkey. 191, p.307.

Kassab OM, Abdelraouf RE, Ibrahim MM, Abo Ellil AA, Abdallah EF (2014). Response of certain varieties of Lentil to deficit irrigation under sandy soils conditions. Middle East Journal of Agriculture Research, 3(4): 1037-1046.

Kowieska A, Petkov K (2003). Lentils (Lens culinaris Medic.) estimation based on macro and microelements content. Zywienie Czowieka i Metabolism, 3(3/4): 1012-1014.

Lal M, Gupta PC, Pandey RK (1988). Response of lentil to different irrigation Schedules. Lens Newsleter, 15(1).

Lardy G, Anderson V (2009). Alternative feeds for ruminants. General concepts and recommendations for using alternative feeds. North Dakota State University Fargo, AS-11(8)2:24.

Lopez-Bellido FJ, Lopez LB, Lopez RJ (2005). Competition, growth and yield of faba bean (Vicia faba L.). Eur. J. Agron. 23,359-378.

Mahmoud EM (2014). Effect of intra-row spacing and seed size on yield and seed quality of faba bean (Vicia faba L) Int. J Agric. Crops Sci. 7(10):665670.

McDonald GK, Hollaway KL, McMurray L (2007). Increasing plant density improves weed competition in lentil (Lens culinaris). Aust. J. Exp. Agric. 47: 48-56.

Metzener H, Rav H, Senger H (1965). Untersuchungen zur synchronisier-barkeit einzelner pigment. Mango-mutanten von Chlorella. Planta, 65: 186-194.

Momoh EJJ , Zhou W (2001). Grow th and yield responses to plant density and stage of transplanting in winter oilseed rape (Brassica napus L.). J. Agron. Crop Sci. 186:253-259.

Mondal MMA, Puteh AB, Malek MA, Kabir AKMR (2013a). Flowering pattern and reproduc tive efficiency in lentil. Legume Res., 36: 153-157.

Mondal MMA, Puteh AB, Malek MA, Roy S, Rafii MY (2013b). Contribution of morphophysiological traits on yield of lentil. Australian J. Crop Sci., 7: 1167-1172.

Mondal MMA, Rahman MA, Akter MB, Fakir MSA (2011b). Effect of foliar application of nitrogen and micronutrients on growth and yield in mung bean. Legume Res., 34: 166-171.

Nazir MS, Joya GS, Ahmad R, Mahmood T (1992). Feasibility and mode of interaction of 
different gram inter-relay cropping system. Pakistan J. Agric. Res. 13(3): 239-244.

Osman AAM, Yagoub SO, Tut OA (2010). Performance of faba beans (Vicia faba L.) cultivars grown in new agro-ecological regions of Sudan (South Soudan). Australian Journal Basic and Applied Science, 4(1):5516-5521.

Ouji S, El-Bok N, Omri BY, Rouaissi M, Mouelhi M, Ben Younes M, Kharrat M (2016). Impact of row spacing and seeding rate on yield components of lentil (Lens culinaris L.). Journal of new Science. 25(2):1138-1144.

Parveen K, Bhuiya MSU (2010). Effect of method of sowing and seed rate on the yield and yield components of lentil. J. Agro for. Environ. 4 (1):155-157.

Raghuvanshi RS, Singh DP (2009). The lentil: botany, production and uses. In W. Erskine, F. J. Muehlbauer, A. Sarker, \& B. Sharma (Eds.), Food Preparation and Use (408-424).

Roysharma RP, Thakur HC, Sharma HM, Mishra SS, Thakur SS (1984). Effect of fertilization and inoculation of pair and late sown pure lentil. Indian J. Agron. 29(4):459-462.

Sarker A, Ersk ine W, Singh M (2003). Regression models for lentil seed and straw yields in Near East. Agric. For. Meteorol. 116: 61-72.

Seyyed GM, Mohamad JS, Mohamad RD (2014). Effect of sowing date and Plant density on yield and yield components of Lentil (Lens culinaris cv.
Sistan). Annual Research \& Review in Biology, 4(1):296-305.

Sharar MS, Ayub M, Nadeem MA, Noori SA (2001). Effect of different row spacing and seeding densities on the growth and yield of gram (Cicer aerietinum L.). Department of Agronomy, Univ. of Agric. Faisalabad, Pakistan. 38(3-4):51-53.

Singh NB, Verma KK (1996). Response of lentil (Lens culinaris) genotypes to spacing in flood-porne area. Indian J. Agron. 41(4):657-658.

Singh ON, Sharma M, Dash R (2003). Effect of seed rate, phosphorus and FYM application on growth and yield of bold seeded lentil. Indian J. pulses Res. 16(2):116-128.

Snedecor GW, Cochran WG (1980). Statistical Methods, $12^{\text {th }}$ ed. Iowa State Univ. Press, Amer. Iowa, USA.

Stoilova T, Pereira MG (1999). Morphological characterization of 120 lentil (Lens culinaris medic) Accession. Lens New sletter. 26(1-2):7-10.

Vites FGJr (1965). Increasing water use efficiency by soil management. In W. H. Pierre, D. Kirkham, j. Pesek and R. Shaw (Eds). "Plant Environment and Efficient Water Use" Amer. Soc. Agron. Madison, Wisc., 259-274.

Wanns MNJ, Denes DH, Barcen RJ (1986). Effects of planting density and design on pod yield of bush snap bean (Phaseolus vulgaris L.). Canadian Journal of Plant Science, 66:669-675.

Wells R (1993). Dynamics of soyabean growth in variable planting patterns. Agron. J., 85: 44-48. 


\section{الملخص العربي}

تأثير نظم الزراعة المختلفة علي انتاجية وكفاءة استخدام الماء لبعض أصناف العدس

أومنية صبحى محمد هاشم ' و محمد عباس ابراهيم '

$$
\text { ' ق قسم بحوث فسيولوجيا المحاصيل - معهد بحوث المحاصيل الحقلية - مركز البحوث الزراعية- الجيزة- مصر. }
$$

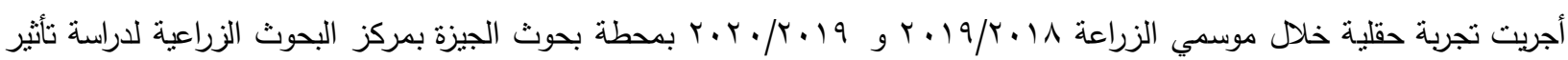



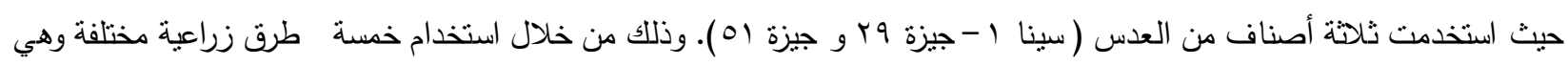

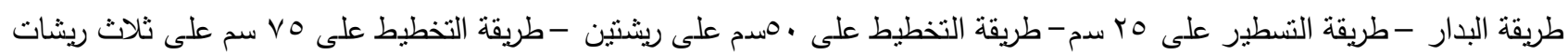

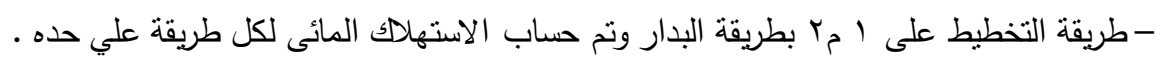

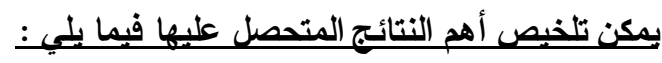

1 - أعطي الصنف جيزة اه أعلا القيم لجميع الصفات المدروسة وكان الافضل في حين أعطي الصنف سينا ا أقل القيم لجميع الصفات المدروسة.

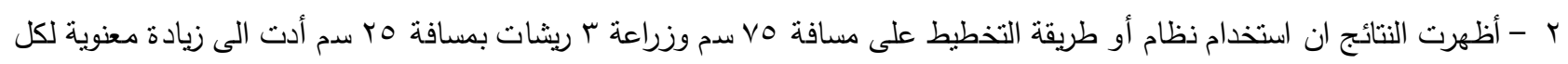

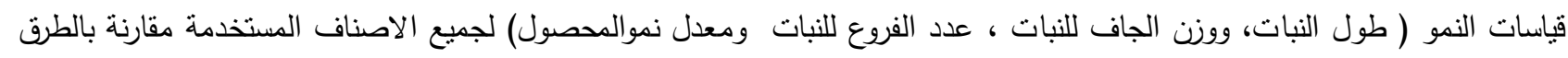

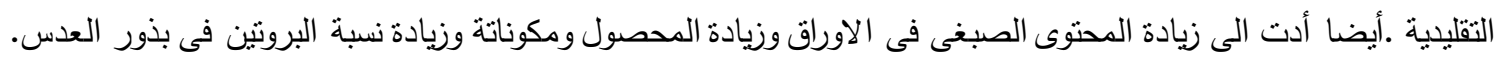

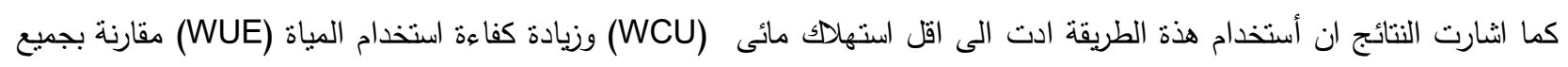
الطرق الاخرى المستخدمة. 